\title{
Catabolic and proinflammatory effects of leptin in chondrocytes are regulated by suppressor of cytokine signaling-3
}

\author{
Anna Koskinen-Kolasa', Katriina Vuolteenaho', Riku Korhonen ${ }^{1}$, Teemu Moilanen ${ }^{1,2}$ and Eeva Moilanen ${ }^{1 *}$
}

\begin{abstract}
Background: Previous studies provide evidence that adipokine leptin increases production of catabolic and proinflammatory factors in chondrocytes and serves as a link between obesity and osteoarthritis (OA). However, the magnitude of the response to leptin treatment varies greatly between chondrocytes from different donor patients. In the present study, we investigated the regulatory role of suppressor of cytokine signaling-3 (SOCS-3) in the leptin-induced responses in OA cartilage.

Methods: Cartilage and synovial fluid samples from 97 patients with OA undergoing knee replacement surgery were collected. Cartilage samples were cultured with leptin $(10 \mu \mathrm{g} / \mathrm{ml})$, and the levels of proinflammatory and catabolic factors in synovial fluid and in the cartilage culture media, and SOCS-3 expression in the cartilage were measured. The role of SOCS-3 in leptin signaling was further studied in $\mathrm{H} 4$ murine chondrocytes by downregulating SOCS-3 with siRNA.

Results: Leptin-induced expression of matrix metalloproteinases MMP-1, MMP-3, MMP-13, interleukin-6 (IL-6), inducible nitric oxide synthase (iNOS) and cyclooxygenase-2 (COX-2) were higher in the cartilage samples with low SOCS-3 expression. Accordingly, downregulation of SOCS-3 by siRNA in $\mathrm{H} 4$ chondrocytes led to enhanced leptin-induced expression of MMP-3, MMP-13, IL-6 and iNOS. Synovial fluid leptin was associated positively, and cartilage SOCS-3 negatively with synovial fluid levels of MMPs in a multivariate model in obese (body mass index (BMI) $>30 \mathrm{~kg} / \mathrm{m}^{2}$ ) but not in non-obese $\left(\mathrm{BMl}<30 \mathrm{~kg} / \mathrm{m}^{2}\right)$ patients.
\end{abstract}

Conclusions: Our results show, for the first time, that SOCS-3 regulates leptin-induced responses in cartilage, and could thus be a future drug target in the treatment or prevention of OA, especially in obese patients.

Keywords: Leptin, Adipokine, SOCS-3, Osteoarthritis, Chondrocytes, Obesity

\section{Background}

Adipokines are cytokine-like hormones produced by adipose tissue and originally discovered to regulate energy metabolism [1,2]. Their role in inflammation and obesity-related disease, such as type 2 diabetes mellitus and cardiovascular disease, and also in rheumatic disease has attracted increasing interest during the past decade. Leptin was first characterized in 1994 [3] and to date it is probably the most studied adipokine. The circulating levels of leptin are closely associated with

\footnotetext{
* Correspondence: eeva.moilanen@uta.fi

${ }^{1}$ The Immunopharmacology Research Group, University of Tampere School

of Medicine and Tampere University Hospital, Tampere, Finland

Full list of author information is available at the end of the article
}

the amount of stored body fat and with body mass index (BMI) [4]. Leptin is, however, not only produced by adipose tissue, but also by several other tissues, including cartilage and other joint tissues [5-7]. Interestingly, synovial fluid leptin levels are also correlated with BMI and leptin expression in chondrocytes is increased in obese individuals with OA $[5,6,8]$. The expression of leptin and its functional receptor $\mathrm{Ob}-\mathrm{Rb}$ is also reported to be increased in chondrocytes in OA, in comparison to healthy chondrocytes [6].

Obesity is a major risk factor for OA [9]. Traditionally obesity has been thought to explain the risk of developing OA due to increased wear-and-tear on weight-bearing joints. However, obesity is also a risk factor for hand OA 
[10], which points to a systemic factor or factors that mediate the obesity-related impact on cartilage. Leptin, with its strong positive association with body fat stores, fits well in this picture; in fact, increasing evidence supports the role of leptin as a significant factor in the pathogenesis of OA. Leptin has been shown to have direct proinflammatory and catabolic effects on cartilage in experimental settings. We and others have previously shown that leptin enhances production of catabolic enzymes, including matrix metalloproteinase 1 (MMP-1), MMP-2, MMP-3, MMP-9, MMP-13, a disintegrin and metalloproteinase with thrombospondin motifs 4 (ADAMTS-4) and ADAMTS-5 and proinflammatory mediators, such as nitric oxide (NO), interleukin 6 (IL-6), IL-1 $\beta$, IL-8 and prostaglandin $\mathrm{E}_{2}\left(\mathrm{PGE}_{2}\right)$ in chondrocytes, synoviocytes and in cartilage [6,11-19]. These findings suggest that leptin is not only a bystander of cartilage breakdown, but an active detrimental factor in the pathogenesis of OA.

According to our experience, cartilage from different donor patients respond to leptin treatment in a quite versatile manner: some of the samples produce large amounts of catabolic/proinflammatory mediators like MMPs, IL-6 and $\mathrm{NO}$ following leptin treatment, while in some samples leptin-induced changes in the production of these factors are very small. Similar wide variation in the response to leptin is also supported by other studies [17]. A study by Pallu et al. showed that primary chondrocytes received from obese patients with OA respond to smaller amounts of leptin to enhance MMP-13 production than chondrocytes obtained from non-obese patients [17], suggesting that obese individuals might be more susceptible to the harmful effects of leptin on cartilage. However, the mechanisms regulating leptin responsiveness in chondrocytes remain unknown.

Suppressor of cytokine signaling 3 (SOCS-3) belongs to SOCS proteins, which are intracellular molecules that have an important function of limiting excessive inflammatory activation of the innate and adaptive immune system [20]. In inflammatory cells SOCS-3 expression is induced by type I and type II cytokine receptors via the JAK-STAT pathway. SOCS-3 binds to the gp130 subunit of those receptors and inhibits the JAK-STAT pathway, thus forming a negative feedback loop to limit cytokine actions [21]. Interestingly, SOCS-3 is also involved in regulating leptin responsiveness in the central nervous system $(\mathrm{CNS})$ [22].

The metabolic function of leptin is to serve as a sensor of body fat stores for the CNS. Elevation of blood leptin due to calorie intake, whether short-term or long-term, in a lean person normally suppresses food intake, whereas decreased leptin levels due to fasting or loss of adipose tissue lead to increased food intake [23]. In obesity however, elevated leptin does not lead to the expected responses in weight control. This is thought to be due to disturbed leptin signaling, also called leptin resistance. Elevated SOCS-3 expression in the CNS is proposed to be the primary mechanism that causes leptin resistance and subsequent failure in controlling food intake in obesity [22]. Consistently, leptindeficient mice develop severe obesity [24], whereas SOCS-3 conditional knockout mice are resistant to diet-induced obesity [25]. In humans, genetic leptin deficiency also causes severe obesity, though leptin and leptinreceptor-related mutations are extremely rare [26].

SOCS-3 is also expressed in cartilage [27-29], and we reported previously that its expression is lower in cartilage from obese patients with $\mathrm{OA}$ than from non-obese patients [8]. That led us to hypothesize that SOCS-3 could be a significant mechanism behind the variable leptin responsiveness in cartilage samples from different donor patients. We addressed the hypothesis by investigating SOCS-3 expression and leptin responsiveness in cartilage samples obtained from 97 patients with OA. In addition, the role of SOCS-3 expression in leptin signaling was studied by downregulating SOCS-3 by siRNA in chondrocyte cultures.

\section{Methods}

\section{Cartilage and cell cultures}

Cartilage and synovial fluid (SF) samples were collected from 97 patients with $\mathrm{OA}$ who were undergoing knee replacement surgery. All patients fulfilled the American College of Rheumatology classification criteria for OA [30]. Cartilage samples were processed for tissue culture as previously described [15]. Cartilage pieces were incubated for 42 hours with or without leptin $(10 \mu \mathrm{g} / \mathrm{ml})$. The concentration of leptin used was chosen based on our previous studies and on existing literature [15, 17-19]. Recombinant human leptin was purchased from $R \& D$ Systems Europe Ltd, Abindgon, UK. Synovial fluid (SF) samples from the corresponding patients were also collected at the beginning of the arthroplasty. The SF samples were centrifuged at $4000 \mathrm{~g}$ at $4{ }^{\circ} \mathrm{C}$ and supernatants were collected and kept at $-70{ }^{\circ} \mathrm{C}$ until assayed.

The immortalized murine $\mathrm{H} 4$ chondrocyte cell line [31], developed in the Laboratory of Experimental Rheumatology, University Medical Center, Nijmegen, The Netherlands, was used in the siRNA experiments. The chondrocytes were cultured at $37^{\circ} \mathrm{C}$ in humidified $5 \%$ carbon dioxide atmosphere in Dulbecco's modified Eagle's medium (DMEM) with L-glutamine and Ham's F-12 medium (1:1) supplemented with $5 \%$ fetal bovine serum (all obtained from Lonza Group Ltd, Basel, Switzerland).

\section{Immunoassays and nitrite measurements}

Concentrations of MMP-1, MMP-3, MMP-13 and IL-6 were determined by immunoassays with commercial reagents according to the protocol provided by the manufacturer (human total MMP-1, human total MMP-3, 
human total MMP-13, mouse total MMP-3 and mouse IL6 ELISA kits were from R\&D Systems; human IL-6 ELISA kit was from Sanquin, Amsterdam, The Netherlands; MMP-1 in SF was determined by Multiplex bead array, Fluorokine ${ }^{\bullet}$ Human MMP Multi Analyte Profiling Base Kit, purchased from R\&D systems). Nitrite, stable metabolite of nitric oxide (NO), was measured in the culture media by the Griess reaction [32]. The cartilage culture media samples were filtered through Amicon Ultra 10-K filters (from Millipore, Cork, Ireland) at 14,000 g prior to the Griess analysis in order to remove large proteins that might interfere with the Griess analysis.

\section{RNA isolation and quantitative reverse transcription/ polymerase chain reaction}

Culture medium was removed at the indicated time points and total RNA of H4 chondrocytes was extracted with GenElute $^{\mathrm{Tu}}$ Mammalian Total RNA Miniprep kit (Sigma-Aldrich, St Louis, MO, USA). Total RNA was treated with DNAse (Fermentas UAB, Vilnius, Lithuania) and reverse-transcribed to cDNA using TaqMan Reverse Transcription reagents and random hexamers (Applied Biosystems, Foster City, CA, USA). cDNA obtained from the RT reaction was diluted 1:20 with RNAse-free water and subjected to quantitative PCR using TaqMan Universal PCR Master Mix and the ABI Prism 7000 Sequence detection system (Applied Biosystems). Primers and probes for SOCS-3, glyceraldehyde-3-phosphate dehydrogenase (GAPDH), iNOS, IL-6 and MMP-13 were obtained from Metabion International AG (Martinsried, Germany). The primer and probe sequences and concentrations (Table 1) were optimized according to the manufacturer's instructions in TaqMan Universal PCR Master Mix Protocol part number 4304449 revision $C$. The expression of mouse MMP-3 mRNA was measured using TagMan Gene Expression Assay (Mm00440295_m1, Applied Biosystems).

PCR reaction parameters were as follows: incubation at $50{ }^{\circ} \mathrm{C}$ for 2 minutes, incubation at $95{ }^{\circ} \mathrm{C}$ for $10 \mathrm{mi}$ nutes, and thereafter 40 cycles of denaturation at $95{ }^{\circ} \mathrm{C}$ for $15 \mathrm{~s}$ and annealing and extension at $60{ }^{\circ} \mathrm{C}$ for $1 \mathrm{mi}-$ nute. Each experimental reaction was performed in duplicate. The relative mRNA levels of SOCS-3, GAPDH, iNOS, IL-6 and MMP-13 were quantified using the standard curve method as described in Applied Biosystems User Bulletin number 2. To calculate the relative expression of MMP-3 mRNA, the $2^{(-\Delta \Delta C T)}$ method [33] was used. According to the method, the cycle threshold $\left(C_{T}\right)$ values for MMP-3 mRNA expression in each sample were normalized to the $C_{T}$ values of GAPDH mRNA in the same sample.

\section{Western blot}

Preparation of cell lysates, SDS-polyacrylamide gel electrophoresis and western blot analysis were carried out as previously described [15]. Mouse monoclonal SOCS-3 antibody (sc-51699), rabbit polyclonal iNOS antibodies (sc-651 and sc-650), goat polyclonal cyclooxygenase-2 (COX-2) antibody (sc-1745) and rabbit polyclonal $\beta$-actin antibody (sc-1615R), and secondary horseradish peroxidase (HRP)-conjugated goat anti-mouse (sc-2005), goat antirabbit (sc-2004) and donkey anti-goat (sc-2020) antibodies were all from Santa Cruz Biotechnology (Santa Cruz, CA, USA). Rabbit polyclonal MMP-13 antibody (ab39012) was from Abcam (Cambridge, MA, USA). Leptin-induced iNOS and COX-2 expression was determined by running the control and leptin-induced samples side by side and the result is given as fold of change in the $\beta$-actinnormalized densitometry value of the leptin-induced versus the control sample.

\section{Downregulation of SOCS-3 expression by siRNA}

$\mathrm{H} 4$ murine chondrocytes were seeded at $1 \times 10^{5}$ cells/well in 24-well plates. Cells were incubated for 24 hours and transfected with SOCS-3 siRNA or with non-targeting control siRNA. On-Target SMART pool SOCS-3-specific siRNA (targeting sequences of GGCUAGGAGACUCGC CUUA, GGACCAAGAACCUACGCAU, CUAAUGAAA CCUCGCAGAU and GAAGGGAGGCAGAUCAACA) and siGENOME Non-Targeting siRNA were used at 100 $\mathrm{nM}$ to transfect the cells using DharmaFECT 1. All transfection reagents were from Thermo Scientific Dharmacon (Lafayette, CO, USA) and transfection was carried out according to the manufacturer's protocol. The experiments were started 48 hours after the transfection by adding leptin $(10 \mu \mathrm{g} / \mathrm{ml})$ (mouse recombinant leptin from $R \& D$ systems) in fresh culture medium.

\section{Statistical analysis}

The chi-square test, unpaired $t$ test and Mann-Whitney test (where appropriate) were used to analyze differences between subgroups of the patients. The Wilcoxon test was used to calculate the significance of leptin-induced effects in the cartilage culture.

To analyze the differences in leptin responsiveness in relation to SOCS-3 expression, the samples on each western blot gel were divided to two equal sized groups (low SOCS-3 or high SOCS-3) according to SOCS-3 expression. Median leptin responses, measured as change in the production of MMP-1, MMP-3, MMP-13, IL-6 and $\mathrm{NO}$ in the leptin-treated versus control sample, and as fold of change in the expression of iNOS and COX-2, were compared between the low SOCS-3 and the high SOCS-3 groups. Possible intergel differences in SOCS-3 expression were controlled by analysis of variance (ANOVA) in which the leptin response variable (e.g., leptin-induced change in production of MMP-1) was set as a dependent variable, western blot gel (1 to 8$)$ as a grouping variable and SOCS-3 
Table 1 Primer and probe sequences for quantitative RT-PCR

\begin{tabular}{|c|c|c|c|}
\hline Gene & Oligunucleotide & Sequence & Conc. (nM) \\
\hline & Forward primer & GCATGGCCTTCCGTGTTC & 300 \\
\hline \multirow[t]{3}{*}{ Mouse GAPDH } & Reverse primer & GATGTCATCATACTTGGCAGGTTT & 300 \\
\hline & Probe & TCGTGGATCTGACGTGCCGCC & 150 \\
\hline & Forward primer & GCGGGCACCTTTCTTATCC & 300 \\
\hline \multirow[t]{3}{*}{ Mouse SOCS-3 } & Reverse primer & AAGCTGCCCCCCTCACA & 300 \\
\hline & Probe & CTCGGACCAGCGCCACTTCTTCA & 150 \\
\hline & Forward primer & CCTGGTACGGGCATTGCT & 300 \\
\hline \multirow[t]{3}{*}{ Mouse iNOS } & Reverse primer & GCTCATGCGGCCTCCTT & 300 \\
\hline & Probe & CAGCAGCGGCTCCATGACTCCC & 150 \\
\hline & Forward primer & TCGGAGGCTTAATTACACATGTTC & 900 \\
\hline \multirow[t]{3}{*}{ Mouse IL-6 } & Reverse primer & CAAGTGCATCATCGTTGTTCATAC & 300 \\
\hline & Probe & CAGAATTGCCATTGCACAACTCTITTCTCA & 200 \\
\hline & Forward primer & TTGTGTTGGAGAGCACTACTTGA & 900 \\
\hline \multirow[t]{2}{*}{ Mouse MMP-13 } & Reverse primer & AACTGTGGAGGTCACTGTAGACTTCTT & 900 \\
\hline & Probe & CATCCTGCGACTCTTGCGGGAATC & 250 \\
\hline
\end{tabular}

SOCS-3 suppressor of cytokine signaling-3, iNOS inducible nitric oxide synthase, IL-6 interleukin-6, MMP-13 matrix metalloproteinase-13, Conc. concentration

expression as a continuous variable as a covariate. Associations were further tested by adjusting for BMI and age.

Correlation between the factors of interest in SF were determined by Pearson's correlation analysis. The associations between MMPs or IL-6 and leptin in SF, and SOCS-3 expression in cartilage were further analyzed by ANOVA modeling, by including the variable of interest (SF MMP-1, MMP-3 or IL-6) as a dependent variable, leptin in SF and SOCS-3 expression in the cartilage as covariates and gel number as a grouping factor. The analysis was done in BMI subgroups (obese, BMI $>30 \mathrm{~kg} / \mathrm{m}^{2}$; non-obese, BMI $<30 \mathrm{~kg} / \mathrm{m}^{2}$ ). Natural logarithms were formed of the leptin response values, SOCS-3 expression levels and SF levels of the measured variables where appropriate in order to have normally distributed variables for the ANOVA modeling and for the correlation analyses.

The data were analyzed by IBM SPSS Statistics 19 (IBM Corporation, NY, USA) and Graph-Pad InStat version 3.00 software (GraphPad Software Inc., San Diego, CA, USA). The results of the siRNA experiments are presented as means (SEM). The statistical significance of these data was calculated by two-way ANOVA with Bonferroni multiple comparisons post-test using Graph-Pad Prism 5 for Windows version 5.04 (GraphPad Software Inc.). Differences were considered statistically significant at $p<0.05$.

\section{Results}

Leptin-induced production of proinflammatory and catabolic factors in osteoarthritic cartilage in relation to clinical factors and SOCS- 3 expression

Patient characteristics and leptin responses in the cultured cartilage across the whole study population and in the obese (BMI $>30 \mathrm{~kg} / \mathrm{m}^{2}$ ) and non-obese subgroups are presented in Table 2. Leptin significantly enhanced the expression of MMP-1, MMP-3, MMP-13, IL-6, iNOS and COX-2 and NO production in OA cartilage ex vivo (Fig. 1). However, there was considerable variation in these responses between the samples from different donor patients (Table 2). There were no statistically significant differences in the leptin responses between obese and non-obese patients (Table 2), and neither did the leptin responses correlate with age, sex or radiographic scaling of OA.

When the patients were divided into subgroups according to SOCS-3 expression in the cartilage, leptin-induced changes in the expression/production of MMP-1, MMP-3, MMP-13, IL-6, NO, iNOS and COX-2 in the cartilage were significantly greater in the samples with low SOCS-3 expression than in the samples with high SOCS-3 expression (Fig. 2). This suggests that the level of SOCS-3 expression determinates the magnitude of leptin-induced inflammatory responses. The results remained statistically significant $(p<0.05)$ for the responses in the expression of MMP-3, MMP-13, IL-6, NO, iNOS and COX-2, and almost significant for response in the expression of MMP-1 $(p=0.10)$ in the ANOVA modeling after controlling for intergel variation, BMI and age.

\section{Synovial fluid levels of MMPs and IL-6 in relation to SF leptin and SOCS-3 expression in cartilage from patients with $O A$}

SF samples were obtained from 90 of the 97 patients. Obese patients had significantly higher SF leptin than non-obese patients, while SF MMP-1 and MMP-3 did not significantly differ between obese and non-obese 
Table 2 Patient characteristics and leptin responses in cartilage cultures in the whole study population and compared across body mass index subgroups

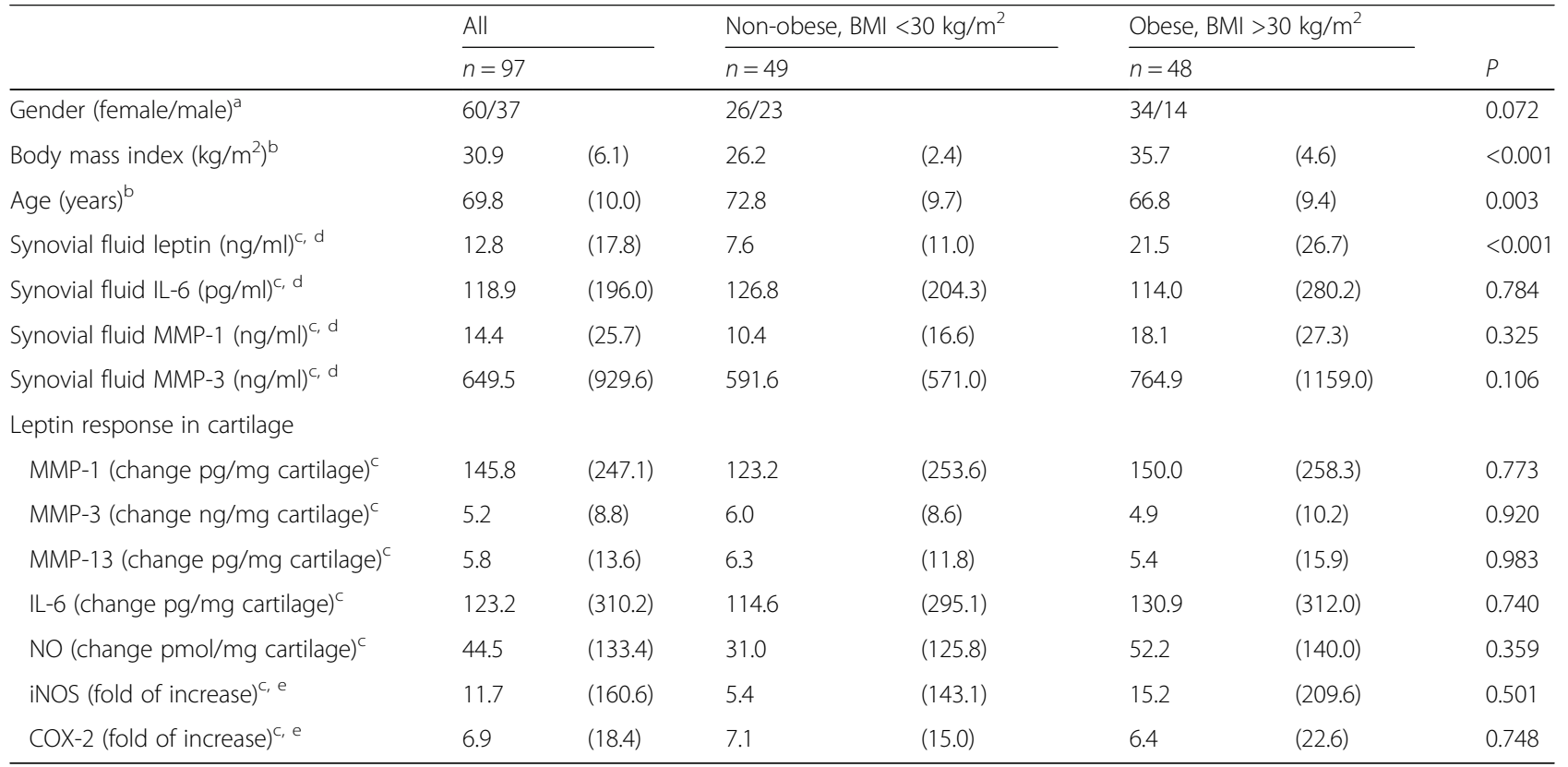

${ }^{a}$ Values are numbers of female/male subjects; $p$ value was calculated for comparison between non-obese and obese subjects using the chi-square test. ${ }^{b}$ Values are mean (SD); $p$ values were calculated for comparison between non-obese and obese subjects using the unpaired $t$ test. 'Values are median (IQR); $p$ values were calculated for comparison between non-obese and obese subjects using the Mann-Whitney test. ${ }^{\mathrm{d}}$ Synovial fluid sample was obtained from 90 patients. ${ }^{\mathrm{e}}$ Numbers of patients (non-obese/obese) in the analysis were $26 / 31$ for inducible nitric oxide synthase (iNOS) and 25/29 for cyclooxygenase-2 (COX-2). MMP matrix metalloproteinase, IL interleukin, NO nitric oxide

patients (Table 2). Leptin correlated positively with MMP1 and with MMP-3 in SF from obese but not from nonobese patients (Fig. 3). In ANOVA modeling, leptin concentrations in SF and SOCS-3 expression in cartilage significantly explained levels of SF MMP-1 and MMP-3 in the obese but not in the non-obese group (Table 3) pointing to obesity-related association of leptin and SOCS-3 in OA pathophysiology. In addition, SF IL-6 levels were explained by SOCS-3 in the obese but not in the non-obese group, while leptin did not significantly explain SF IL-6 levels in either of the BMI subgroups (Table 3).

\section{SOCS-3 modulates leptin responses in chondrocytes}

In order to investigate further the role of SOCS-3 in the regulation of leptin-induced responses in chondrocytes, we used siRNA to downregulate SOCS-3 in the H4 chondrocyte cell line. H4 chondrocytes expressed SOCS-3 mRNA at relatively high levels and it was reduced by approximately $80 \%$ in the SOCS-3-siRNA-treated cells when compared to the cells transfected with control siRNA. Leptin had a clear effect on inducing MMP-3, MMP-13, IL-6 and iNOS expression in the SOCS-3deficient cells, whereas in the control siRNA-treated cells leptin did not have any statistically significant effect on the production of these factors (Fig. 4), confirming that SOCS-3 negatively regulates leptin-induced proinflammatory responses in chondrocytes.

\section{Discussion}

Leptin has been shown to have detrimental effects on cartilage metabolism in several studies [6, 11-19]. However, considerable variation in leptin responsiveness between cartilage/chondrocytes from different patients has been observed. Our present results indicate that a significant mechanism behind the differential leptin responsiveness could be SOCS-3.

SOCS-3 is a known negative regulator of inflammatory signals [20]. Its role in controlling the effects of leptin in chondrocytes has not been previously investigated, but it has been reported to regulate the responses of leptin in the CNS [22]. In the present study we show, for the first time, that SOCS-3 regulates the proinflammatory and catabolic effects of leptin in chondrocytes. This was demonstrated as greater leptin responsiveness in cartilage explants with low SOCS-3 expression in comparison to lower leptin responsiveness in the explants with high SOCS-3 expression. The causality of this association was illustrated by downregulation of SOCS-3 by siRNA in the chondrocyte cell line, which led to increased leptininduced expression of proinflammatory and catabolic genes. In addition, SF leptin levels were shown to be positively associated, and cartilage SOCS-3 expression negatively associated with SF MMP levels in obese, but not in non-obese patients with OA. This points to dysregulation of the leptin-SOCS-3 axis, especially in obese 

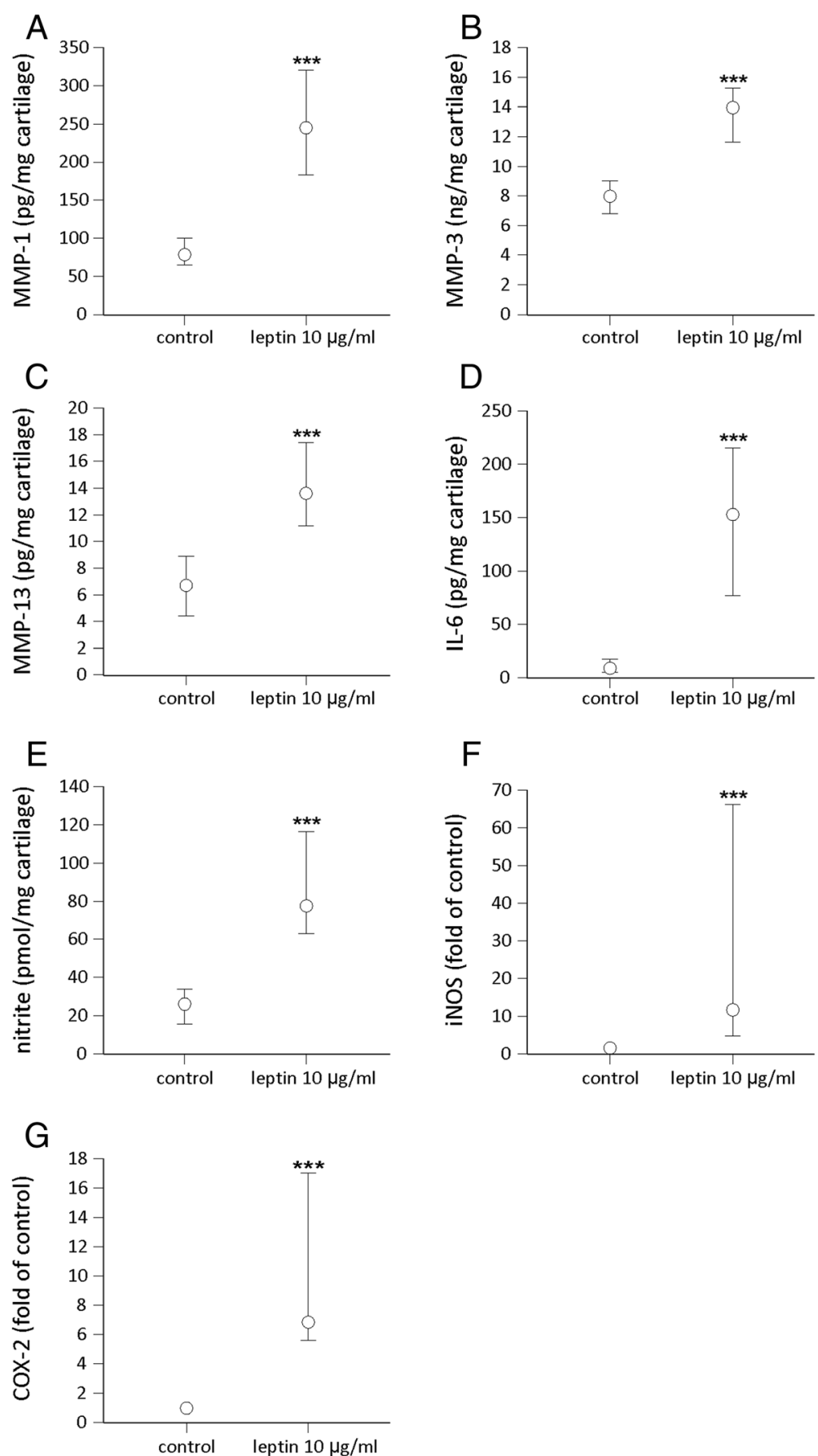

Fig. 1 Effect of leptin on the production of matrix metalloproteinase-1 (MMP-1) (a), MMP-3 (b), MMP-13 (c), interleukin-6 (IL-6) (d), nitric oxide (NO) (e) and on the expression of inducible nitric oxide synthase (iNOS) (f) and cyclooxygenase-2 (COX-2) (g) in cartilage from patients with osteoarthritis (OA). Cartilage samples from 97 patients with OA were cultured with and without leptin (10 $\mathrm{mg} / \mathrm{ml})$ for 42 hours. Concentrations of MMP-1, MMP-3, MMP-13 and IL-6 were measured by ELISA; NO production was determined as its metabolite nitrite by the Griess reaction and iNOS and COX-2 proteins by western blotting. The circles represent the medians. The whiskers represent $95 \%$ confidence interval of the median. Statistical significance was calculated using the Wilcoxon test; ${ }^{* *} p<0.001$

individuals, and to a possible obesity-related pathogenic mechanism in OA.

In the present study we observed a positive association between leptin levels and matrix metalloproteinases in
SF that was only present in the obese patients with OA. However, obesity did not explain the differential leptin responsiveness in the cartilage culture experiments, unlike in the study by Pallu et al. where greater leptin 

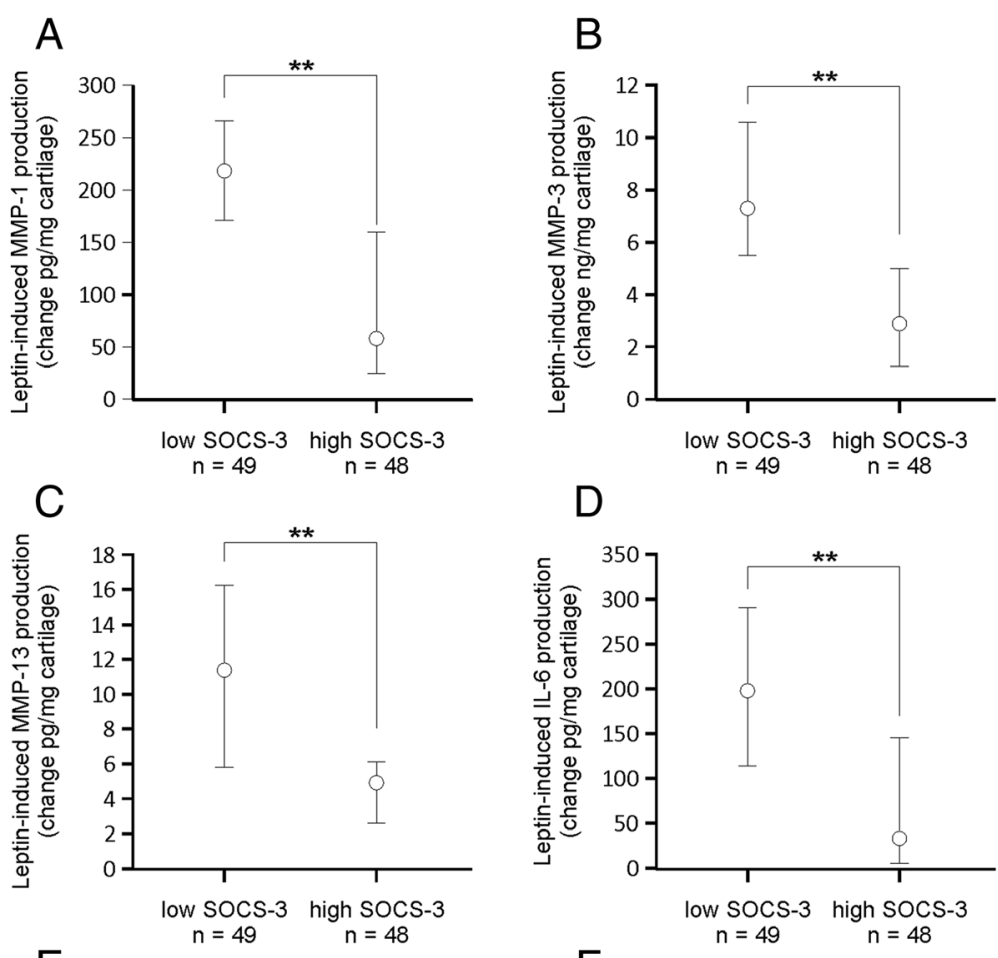

$\mathrm{D}$
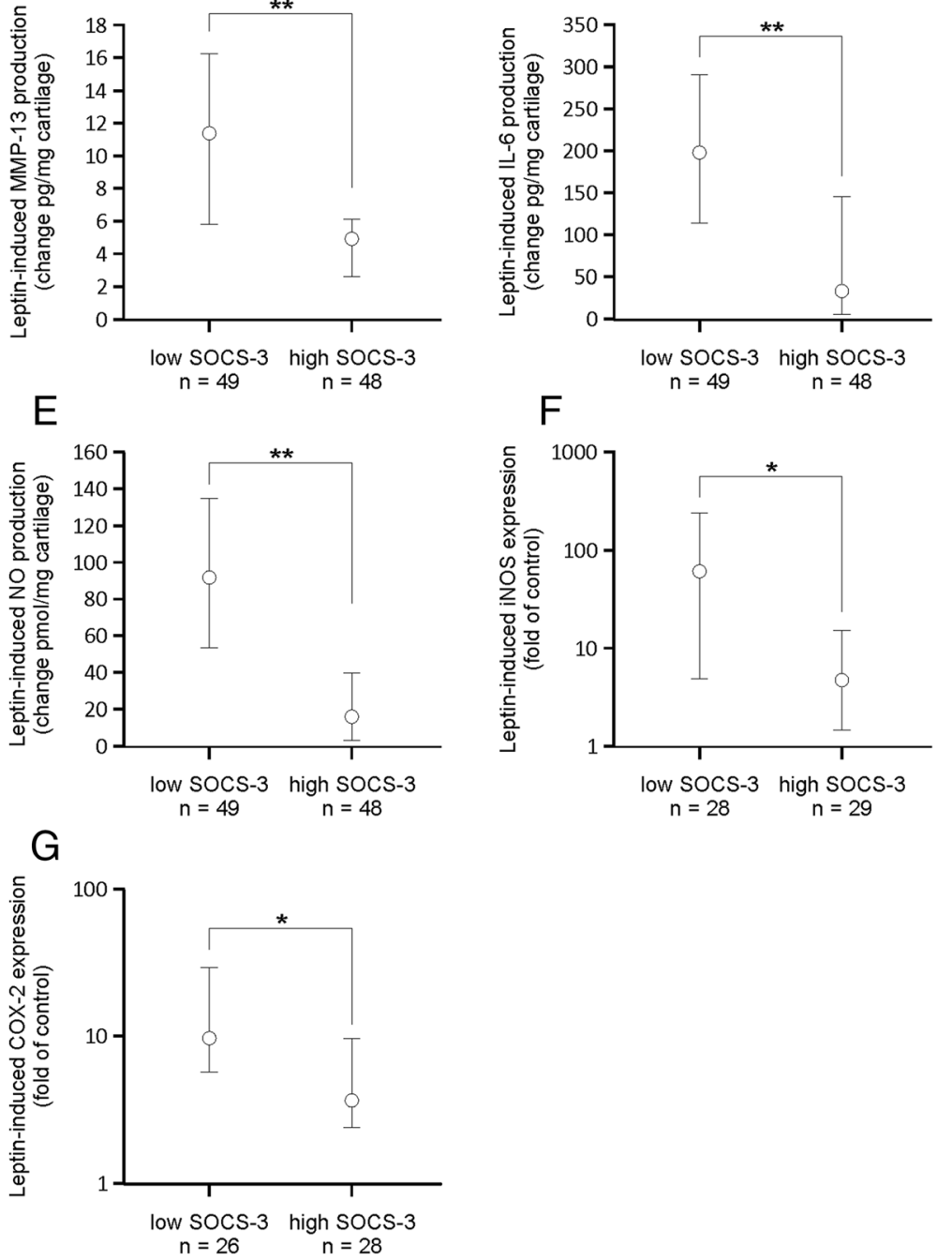

Fig. 2 Leptin-induced production/expression of matrix metalloproteinase-1 (MMP-1) (a), MMP-3 (b), MMP-13 (c), interleukin-6 (IL-6) (d), nitric oxide (NO) (e), inducible nitric oxide synthase (iNOS) (f) and cyclooxygenase-2 (COX-2) (g) in cartilage from patients with osteoarthritis (OA) in subgroups stratified by suppressor of cytokine signaling-3 (SOCS-3) expression in the non-treated cartilage. Human osteoarthritic cartilage was cultured with leptin $(10 \mu \mathrm{g} / \mathrm{ml})$ for 42 hours. Concentrations of MMP-1, MMP-3, MMP-13 and IL-6 were measured by ELISA, NO was determined as its metabolite nitrite by the Griess reaction and INOS and COX-2 proteins were analyzed by western blotting. The circles represent the median change in the leptin-induced effects. The whiskers represent the $95 \%$ confidence interval of the median. Numbers of patients from whom the cartilage samples were collected are indicated. Statistical significance was calculated using the Mann-Whitney test; ${ }^{*} p<0.05,{ }^{* *} p<0.01$ 

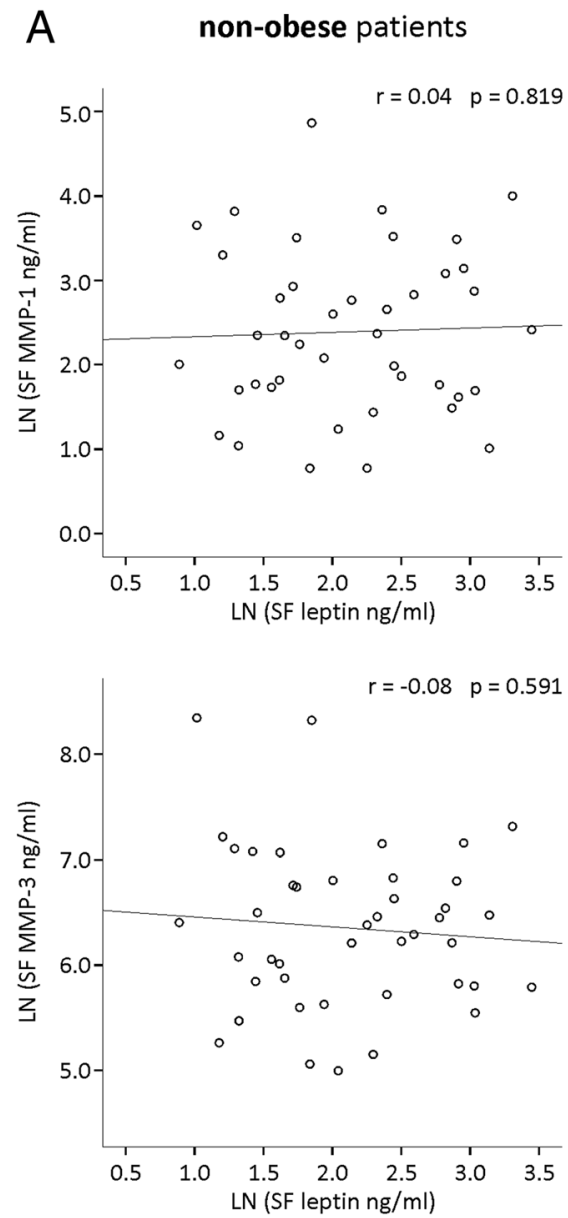

B obese patients
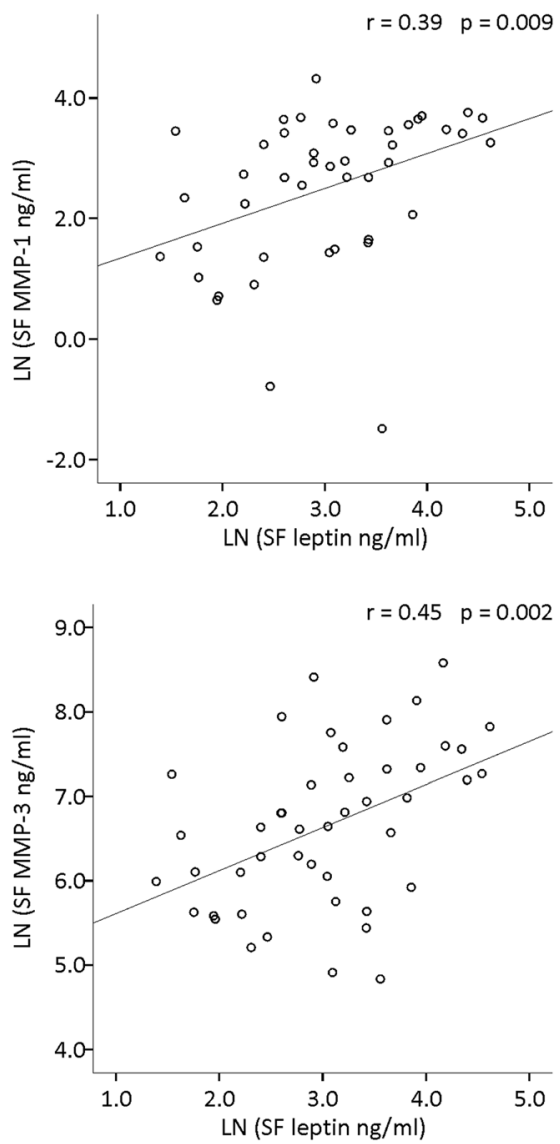

Fig. 3 Correlation between leptin and matrix metalloproteinase-1 (MMP-1) and MMP-3 in non-obese (a) and obese (b) patients with osteoarthritis Leptin and MMPs were measured in synovial fluid (SF) by immunoassay. Natural logarithms (LN) were formed of the SF levels of leptin and MMPs in order to have normally distributed variables for the Pearson correlation analysis. Correlation coefficients $(r)$ and $p$ values are indicated. Samples were collected from 90 patients (non-obese, $\mathrm{BMl}<30 \mathrm{~kg} / \mathrm{m}^{2}, n=44$; obese, BMI $>30 \mathrm{~kg} / \mathrm{m}^{2}, n=46$ )

Table 3 Associations between interleukin-6 (IL-6), matrix metalloproteinase-1 (MMP-1), MMP-3 and leptin in synovial fluid and suppressor of cytokine signaling-3 (SOCS-3) expression in cartilage from non-obese and obese patients with osteoarthritis

\begin{tabular}{|c|c|c|c|c|c|}
\hline \multirow[b]{2}{*}{ Dependent variable } & \multirow[b]{2}{*}{ Covariates } & \multicolumn{2}{|c|}{ Non-obese, $\mathrm{BMI}<30 \mathrm{~kg} / \mathrm{m}^{2}$} & \multicolumn{2}{|c|}{ Obese, $\mathrm{BMI}>30 \mathrm{~kg} / \mathrm{m}^{2}$} \\
\hline & & $R^{2}$ adjusted & P & $\overline{R^{2} \text { adjusted }}$ & $P$ \\
\hline \multirow[t]{3}{*}{ LN (SF MMP-1) } & & 0.15 & & 0.30 & \\
\hline & LN SOCS-3 & & 0.818 & & 0.007 \\
\hline & LN (SF leptin) & & 0.884 & & 0.023 \\
\hline \multirow[t]{3}{*}{ LN (SF MMP-3) } & & 0.03 & & 0.27 & \\
\hline & LN SOCS-3 & & 0.608 & & 0.004 \\
\hline & LN (SF leptin) & & 0.733 & & 0.015 \\
\hline \multirow[t]{3}{*}{ LN (SF IL-6) } & & -0.05 & & 0.20 & \\
\hline & LN SOCS-3 & & 0.945 & & 0.003 \\
\hline & LN (SF leptin) & & 0.808 & & 0.466 \\
\hline
\end{tabular}

$P$ values are calculated for covariates in analysis of variance modeling. The model is controlled for intergel variation in SOCS-3 expression levels. Analysis was performed in body mass index (BMI) subgroups. Natural logarithms (LN) were formed where appropriate. SF synovial fluid 

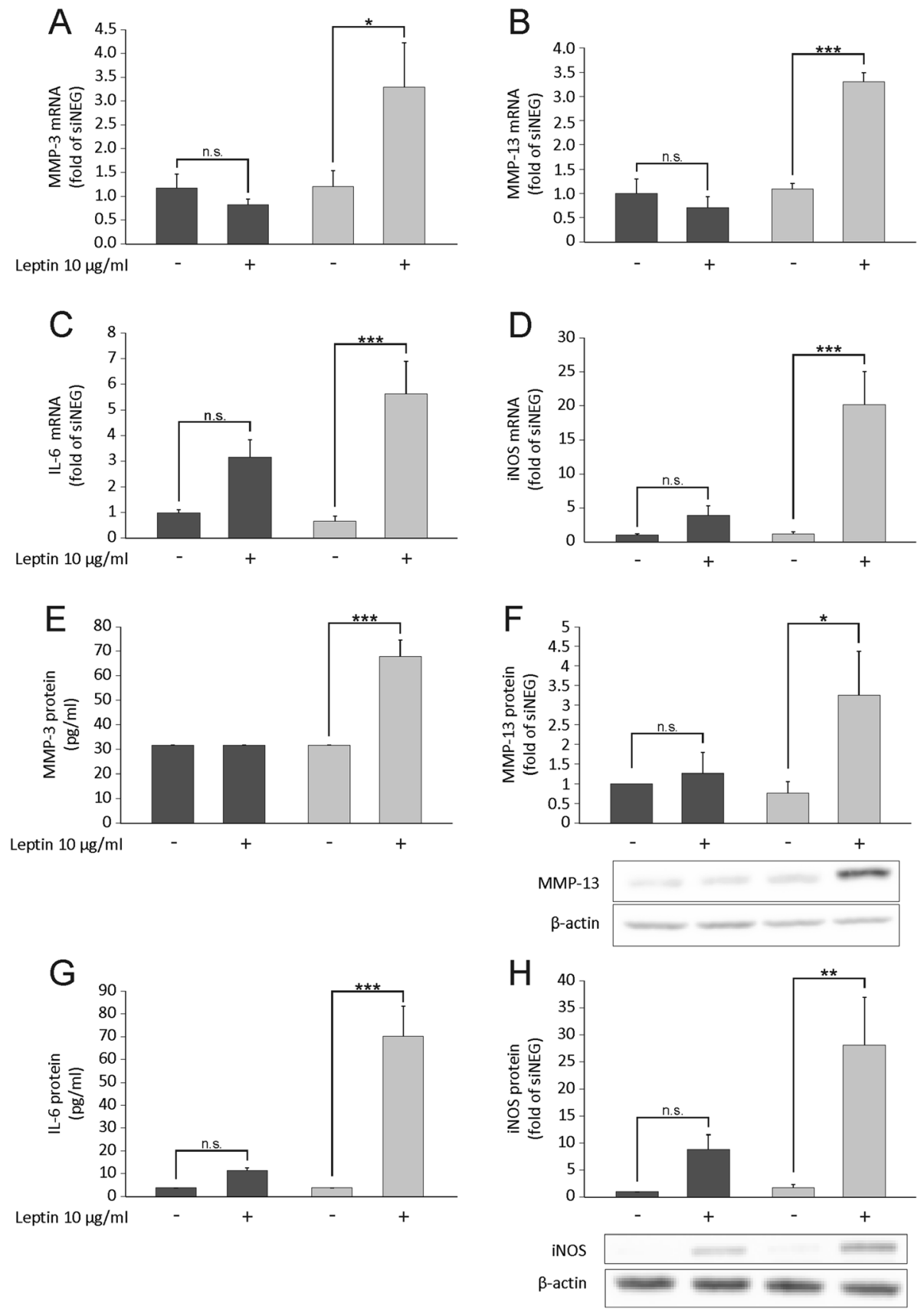

Fig. 4 The effect of silencing of suppressor of cytokine signaling-3 (SOCS-3) by siRNA on leptin-induced expression of matrix metalloproteinase-3 $(M M P-3)(\mathbf{a}, \mathbf{e}), M M P-13(\mathbf{b}, \mathbf{f})$, interleukin-6 (IL-6) $(\mathbf{c}, \mathbf{g})$ and inducible nitric oxide synthase (iNOS) (d, $\mathbf{h})$ in H4 murine chondrocytes. The cells were transfected with SOCS-3 siRNA or non-targeting siRNA (siNEG) and treated with leptin $(10 \mu \mathrm{g} / \mathrm{ml})$ for $4(\mathbf{c}, \mathbf{d}), 8(\mathbf{a}, \mathbf{b}, \mathbf{h})$ or 24 (e-g) hours. mRNA expression (a-d) was determined by quantitative RT-PCR, the levels of MMP-3 (e) and IL-6 (g) in the culture media supernatants by ELISA, and MMP-13 (f) and INOS (h) expression in the chondrocyte lysates by western blotting. Results are expressed as means \pm SEM; $n=6$ (a-e and $\mathbf{g})$ and $n=3(\mathbf{f}, \mathbf{h})$. MMP-3 protein level in siNEG and in non-treated SOCS-3 siRNA samples was below the detection limit and is set as half of the lowest standard. Representative bands of the western blots are shown. Statistical analysis was carried out by two-way analysis of variance with Bonferroni multiple comparisons post hoc test; ${ }^{*} p<0.05,{ }^{* *} p<0.01,{ }^{* *} p<0.001$. n.s. not significant 
response in MMP-13 mRNA expression in primary chondrocytes obtained from obese in comparison with non-obese patients with OA was reported [17]. The differences between these two studies could be explained by differential experimental conditions. Instead of primary chondrocytes we used pieces of cartilage, which provides a more natural environment for the chondrocytes in the culture. In addition, we used higher concentrations of leptin to ensure adequate penetration of leptin into the cartilage explant tissue. Of note, in the experimental studies, all cartilage explants were treated with the same leptin concentration in the culture in contrast to the in vivo situation where leptin concentration in SF is highly relative to BMI $[5,6,8]$.

Other factors that might affect leptin responsiveness in vivo and that could contribute to the differential leptin responsiveness in cartilage from individual donors as seen in the present study, include functional leptin receptor $(\mathrm{Ob}-\mathrm{Rb})$ expression in chondrocytes and the amount of soluble leptin reseptor (sOb-R) in SF or in environment surrounding the chondrocytes. We previously reported that the level of soluble leptin receptor, that is thought to bind active leptin, is decreased in SF in obese subjects [8]. The expression of Ob-Rb has been shown to be unaffected in obesity $[6,17]$, whereas it has been reported to be increased in severely damaged cartilage $[5,6]$. In vivo, all of these mechanisms are likely to contribute to the quantity of leptin-evoked effects, obesity seemingly favoring enhanced responses in many ways.

Leptin binding to its receptor leads to activation of multiple intracellular pathways including the Janus kinase-2 (JAK2)-STAT3, mitogen-activated protein kinase (MAPK), nuclear factor (NF)-kB and PI3K/Akt pathways, all of which have been shown to be involved in the leptin-induced production of proinflammatory factors by chondrocytes [11-15, 18, 19]. SOCS-3 has been shown to inhibit not only the STAT3 pathway, but also the extracellular signal-related kinase (Erk) $1 / 2$ and NF-kB pathways [34, 35], providing a possible mechanistic explanation for how leptin-induced responses could be modulated by SOCS-3 in chondrocytes. Interestingly Pallu et al. report higher leptin-induced activation of STAT3 in chondrocytes from obese than from nonobese patients [17], which could be a consequence of decreased SOCS-3 expression, and may explain the differential leptin responsiveness in obese individuals observed in their study. However, the involvement of SOCS-3 expression in explaining increased STAT3 activation in chondrocytes needs to be further studied.

According to the data in the present study, SOCS-3 appears to be a cartilage-protective factor in $\mathrm{OA}$, as low SOCS-3 expression was associated with enhanced proinflammatory and catabolic effects of leptin. Here we investigated the role of SOCS-3 only in leptin signaling, but its role in OA may be much wider, as it can be involved in the regulation of the inflammatory responses induced by a variety of cytokines in multiple cell types in the joint [27, 36]. To our knowledge, only a few groups have previously investigated SOCS-3 expression in chondrocytes. Van de loo et al. reported that SOCS-3 overexpression inhibits lipopolysachharide (LPS)-induced NO production in chondrocytes [29] supporting the idea that SOCS-3 has a similar function in chondrocytes as in white blood cells, that is, to limit excessive inflammatory response. The role of SOCS-3 in arthritis has been studied in animal models by a few groups and the existing data support the idea that SOCS-3 has a protective role in arthritis.

In a study by Shouda et al. SOCS-3 overexpression by intra-articular adenoviral gene transfer prevented the development of collagen-induced arthritis in mice [37]. Veenbergen et al. reported similar results [38]. In their study SOCS-3 was delivered into the animals also by adenovirus gene transfer, but intravenously. Conversely, in a study by Wong et al. conditional deletion of SOCS-3 in the hematopoietic and endothelial cell compartment led to particularly severe arthritis in a mouse model [39], supporting the importance of SOCS-3 as a negative regulator of inflammation. In the first two studies mentioned $[37,38]$ the target cells of SOCS-3 overexpression were supposed to be synoviocytes, antigen-presenting cells and possibly also B and T lymphocytes. In OA, chondrocytes are thought to be the central cell population that produces pathogenic factors; however, synoviocytes and inflammatory cells are also assumed to contribute to the inflammatory process. The present results indicate that SOCS-3 also modulates the inflammatory response in chondrocytes and thus, could be a promising drug target in the prevention/treatment of OA.

It is unclear, what explains the differential SOCS-3 expression in the cartilage from patients with $\mathrm{OA}$ in the present study. Cytokines including IL-1, IL-6, IFN- $\gamma$ and TNF- $\alpha$ are all known inducers of SOCS-3 [40] and can be also found in the SF of affected joints in OA [41]. Interestingly, anti-inflammatory cytokine IL-10, statins and drugs that elevate cAMP are also known to induce SOCS-3 expression [40]. As it is induced by multiple factors, it is likely that the level of SOCS-3 expression in chondrocytes is defined by a complex net effect of proinflammatory and anti-inflammatory factors. SOCS-3 expression has been reported to be elevated in chondrocytes from patients with OA and RA in comparison to patients with femoral neck fracture without arthritis [29]; this is consistent with previous findings in other tissues, suggesting that SOCS-3 expression is elevated at the sites of inflammation, possibly as a regulatory mechanism to limit excessive inflammation response [42]. It is also possible that SOCS-3 expression would be affected by genetic polymorphisms in SOCS-3. There are two single nucleotide 
polymorphisms (SNPs) previously described in human SOCS-3, one in the promoter region and another in the exon 1 of SOCS-3. However, in a large case-control study by Hölter et al. [43] these SNPs were found to have no effect on the expression/function of SOCS-3 and there were no differences in the frequency of these SNPs between overweight and underweight individuals.

In the present study leptin was positively associated and SOCS-3 was negatively associated with MMP levels in SF in obese but not in non-obese patients with OA, further confirming the importance of the leptin-SOCS-3 axis in cartilage metabolism and its possible significance in obesity-induced OA. Obesity is a significant risk factor for OA. In a Finnish population-based long-term follow-up study, a sevenfold risk of developing knee OA was reported in obese individuals with BMI $>30 \mathrm{~kg} / \mathrm{m}^{2}$, as compared with subjects with BMI $<25 \mathrm{~kg} / \mathrm{m}^{2}$ [44]. Several epidemiological and cross-sectional studies have investigated the association between leptin and the prevalence/incidence of $\mathrm{OA}$, pain in $\mathrm{OA}$ and structural changes in the cartilage in OA [45-55].

Leptin is linked to OA in many of those studies but the results are partly conflicting. The differences might arise from the research frame and also from difficulty in differentiating the impact of body fat stores and leptin by statistical means, as these factors correlate strongly with one another. Karvonen-Gutirrez et al. used sophisticated statistical analysis, by adjusting their data for residuals from the regression of leptin on BMI, aiming to control their data for factors other than the metabolic component of BMI [54]. Interestingly, in their population-based study leptin predicted cartilage defects, as detected by magnetic resonance imaging (MRI) at the 10-year follow-up [54].

Another way to separate the effect of obesity and leptin was attempted by the use of leptin-deficient animals. In fact, Griffin et al. showed that leptin-deficient C57BL mice with diet-induced obesity did not develop OA like the corresponding wild-type mice, suggesting that obesity without increased leptin does not lead to OA [56]. In the present study we analyzed patients with OA in BMI subgroups (obese and non-obese) and interestingly, leptin correlated with MMP enzymes in SF only in the obese patients. This finding further supports the role of leptin in connecting obesity and OA. OA is a heterogenic disease with many known risk factors and it is likely that cartilage destruction in non-obese individuals is driven by pathogenic factors other than leptin. We also showed here that SOCS-3 expression in cartilage is negatively associated with SF levels of IL-6 and MMPs, and this was also seen only in the obese group, suggesting that SOCS-3 expression might be inadequate in the cartilage of obese individuals. Our previous finding that SOCS-3 expression in OA cartilage is decreased in obese compared to nonobese patients [8] supports this idea.

\section{Conclusions}

As a summary of this work, we demonstrated that SOCS-3 negatively modulates the pathogenic effects of leptin in chondrocytes. In addition, SOCS-3 expression in cartilage was negatively associated and synovial fluid leptin levels positively associated with synovial fluid MMP concentrations in obese, but not in non-obese patients with OA. This supports the harmful role of leptin and meaningful regulator role of SOCS-3 in obesity-related OA. Considering these factors in future studies could help to recognize new targets in the treatment and prevention of obesity-related OA.

\section{Abbreviations}

ADAMTS: a disintegrin and metalloproteinase with thrombospondin motifs; ANOVA: analysis of variance; BMI: body mass index; cDNA: complementary DNA; CNS: central nervous system; COX-2: cyclooxygenase-2; $\mathrm{C}_{\mathrm{T}}$ : cycle threshold; DMEM: Dulbecco's modified Eagle's medium; ELISA: enzyme-linked immunosorbent assay; Erk1/2: extracellular signal-regulated kinase 1/2; GAPDH: glyceraldehyde-3-phosphate dehydrogenase; gp130: glycoprotein 130; IFN: interferon; IL: interleukin; iNOS: inducible nitric oxide synthase; JAK: Janus kinase; LN: natural logarithm; LPS: lipopolysaccharide; MAPK: mitogen-activated protein kinase; MMP: matrix metalloproteinase; MRI: magnetic resonance imaging; mRNA: messenger RNA; NF-kB: nuclear factor kappa B; NO: nitric oxide; $\mathrm{OA}$ : osteoarthritis; Ob-Rb: functional leptin receptor; PCR: polymerase chain reaction; $\mathrm{PGE}_{2}$ : prostaglandin $\mathrm{E}_{2}$; PI3K: phosphoinositide 3-kinase; SEM: standard error of the mean; SF: synovial fluid; siRNA: small interfering RNA; SNP: single nucleotide polymorphism; sOb-R: soluble leptin receptor; SOCS-3: suppressor of cytokine signaling-3; STAT: a signal transducer and activator of transcription; TNF: tumor necrosis factor

\section{Acknowledgements}

The excellent technical assistance of Meiju Kukkonen, Marja-Leena Lampén, Marja Jousimies, Jan Koski, Mirva Järvelä-Stolting, Nea Bister and Ella Lehto, and the skillful secretarial help of Heli Määttä are greatly acknowledged. Statistician Heini Huhtala is warmly thanked for her advice on the statistical analysis.

\section{Funding}

This study was financially supported by The Academy of Finland, the Competitive Research Funding of the Pirkanmaa Hospital District, Päivikki and Sakari Sohlberg Foundation, the Orion-Farmos Research Foundation and the Scandinavian Rheumatology Research Foundation. AKK is a graduate student in the Tampere Graduate Program in Biomedicine and Biotechnology.

\section{Authors' contributions}

AKK was involved in the conception and design of the study, the laboratory analyses, calculation of the results and interpretation of the data and she drafted the manuscript. KV was involved in the conception and design of the study, the laboratory analyses, calculation of the results, interpretation of the data and drafting the manuscript. RK was involved in the conception and design of the siRNA experiments, calculation of the results, interpretation of the results and revising the manuscript. TM was involved in the conception and design of the study, selecting the patients, acquiring the patient samples, interpretation of the data and revising the manuscript. EM was involved in the conception and design of the study, interpretation of the data and drafting the manuscript. All authors approved the final version of the manuscript.

\section{Competing interests}

The authors declare that they have no competing interests.

\section{Consent for publication}

Not applicable.

Ethics approval and consent to participate

All patients gave their informed consent to the study. The study was approved by the Ethics Committee of Tampere University Hospital and it was carried out in accordance with the Declaration of Helsinki. 


\section{Author details}

${ }^{1}$ The Immunopharmacology Research Group, University of Tampere School of Medicine and Tampere University Hospital, Tampere, Finland. ${ }^{2}$ Coxa Hospital for Joint Replacement, Tampere, Finland.

\section{Received: 4 May 2016 Accepted: 5 September 2016}

Published online: 03 October 2016

\section{References}

1. Vuolteenaho K, Koskinen A, Moilanen E. Leptin - a link between obesity and osteoarthritis. Applications for prevention and treatment. Basic Clin Pharmacol Toxicol. 2014;114:103-8.

2. Scotece M, Conde J, Vuolteenaho K, Koskinen A, Lopez V, Gomez-Reino J, Lago F, Moilanen E, Gualillo O. Adipokines as drug targets in joint and bone disease. Drug Discov Today. 2014;19:241-58.

3. Zhang Y, Proenca R, Maffei M, Barone M, Leopold L, Friedman JM. Positional cloning of the mouse obese gene and its human homologue. Nature. 1994, 372:425-32.

4. Considine RV, Sinha MK, Heiman ML, Kriauciunas A, Stephens TW, Nyce MR, Ohannesian JP, Marco CC, McKee $\amalg$, Bauer TL. Serum immunoreactive-leptin concentrations in normal-weight and obese humans. N Engl J Med. 1996;334: 292-5.

5. Dumond H, Presle N, Terlain B, Mainard D, Loeuille D, Netter P, Pottie P. Evidence for a key role of leptin in osteoarthritis. Arthritis Rheum. 2003;48: 3118-29.

6. Simopoulou T, Malizos KN, Iliopoulos D, Stefanou N, Papatheodorou L, loannou M, Tsezou A. Differential expression of leptin and leptin's receptor isoform (Ob-Rb) mRNA between advanced and minimally affected osteoarthritic cartilage; effect on cartilage metabolism. Osteoarthritis Cartilage. 2007;15:872-83.

7. Iliopoulos D, Malizos KN, Tsezou A. Epigenetic regulation of leptin affects MMP-13 expression in osteoarthritic chondrocytes: possible molecular target for osteoarthritis therapeutic intervention. Ann Rheum Dis. 2007;66:1616-21.

8. Vuolteenaho K, Koskinen A, Moilanen T, Moilanen E. Leptin levels are increased and its negative regulators, SOCS-3 and SOb-R are decreased in obese patients with osteoarthritis: a link between obesity and osteoarthritis. Ann Rheum Dis. 2012;71:1912-3.

9. Blagojevic M, Jinks C, Jeffery A, Jordan KP. Risk factors for onset of osteoarthritis of the knee in older adults: a systematic review and metaanalysis. Osteoarthritis Cartilage. 2010;18:24-33.

10. Kloppenburg M, Kwok WY. Hand osteoarthritis-a heterogeneous disorder. Nat Rev Rheumatol. 2011:8:22-31.

11. Otero M, Gomez Reino JJ, Gualillo O. Synergistic induction of nitric oxide synthase type II: in vitro effect of leptin and interferon-gamma in human chondrocytes and ATDC5 chondrogenic cells. Arthritis Rheum. 2003:48:404-9.

12. Otero M, Lago R, Lago F, Reino JJ, Gualillo O. Signalling pathway involved in nitric oxide synthase type II activation in chondrocytes: synergistic effect of leptin with interleukin-1. Arthritis Res Ther. 2005;7:R581-91.

13. Otero M, Lago R, Gomez R, Lago F, Gomez-Reino JJ, Gualillo O. Phosphatidylinositol 3-kinase, MEK-1 and p38 mediate leptin/interferon-gamma synergistic NOS type II induction in chondrocytes. Life Sci. 2007;81:1452-60.

14. Tong KM, Shieh DC, Chen CP, Tzeng CY, Wang SP, Huang KC, Chiu YC, Fong YC, Tang CH. Leptin induces IL-8 expression via leptin receptor, IRS-1, PI3K, Akt cascade and promotion of NF-kappaB/p300 binding in human synovial fibroblasts. Cell Signal. 2008:20:1478-88.

15. Vuolteenaho K, Koskinen A, Kukkonen M, Nieminen R, Päivärinta U, Moilanen T, Moilanen E. Leptin enhances synthesis of proinflammatory mediators in human osteoarthritic cartilage-mediator role of $\mathrm{NO}$ in leptininduced PGE2, IL-6, and IL-8 production. Mediators Inflamm. 2009;2009: 345838.

16. Bao JP, Chen WP, Feng J, Hu PF, Shi ZL, Wu LD. Leptin plays a catabolic role on articular cartilage. Mol Biol Rep. 2010;37:3265-72.

17. Pallu S, Francin PJ, Guillaume C, Gegout-Pottie P, Netter P, Mainard D, Terlain B, Presle N. Obesity affects the chondrocyte responsiveness to leptin in patients with osteoarthritis. Arthritis Res Ther. 2010;12:R112.

18. Koskinen A, Vuolteenaho K, Nieminen R, Moilanen T, Moilanen E. Leptin enhances MMP-1, MMP-3 and MMP-13 production in human osteoarthritic cartilage and correlates with MMP-1 and MMP-3 in synovial fluid from OA patients. Clin Exp Rheumatol. 2011;29:57-64.

19. Hui W, Litherland GJ, Elias MS, Kitson Gl, Cawston TE, Rowan AD, Young DA. Leptin produced by joint white adipose tissue induces cartilage degradation via upregulation and activation of matrix metalloproteinases. Ann Rheum Dis. 2012;71:455-62.

20. O'Shea JJ, Murray PJ. Cytokine signaling modules in inflammatory responses. Immunity. 2008;28:477-87.

21. Yoshimura A, Naka T, Kubo M. SOCS proteins, cytokine signalling and immune regulation. Nat Rev Immunol. 2007:7:454-65.

22. St-Pierre J, Tremblay ML. Modulation of leptin resistance by protein tyrosine phosphatases. Cell Metab. 2012;15:292-7.

23. Park HK, Ahima RS. Leptin signaling. F1000Prime Rep. 2014;6:73. doi:10. 12703/P6-73.

24. Myers Jr MG. Leptin receptor signaling and the regulation of mammalian physiology. Recent Prog Horm Res. 2004;59:287-304.

25. Mori H, Hanada R, Hanada T, Aki D, Mashima R, Nishinakamura H, Torisu T, Chien KR, Yasukawa H, Yoshimura A. Socs3 deficiency in the brain elevates leptin sensitivity and confers resistance to diet-induced obesity. Nat Med. 2004;10:739-43.

26. Dubern B, Clement K. Leptin and leptin receptor-related monogenic obesity. Biochimie. 2012;94:2111-5.

27. Smeets RL, Veenbergen S, Arntz OJ, Bennink MB, Joosten LA, van den Berg $W B$, van de Loo FA. A novel role for suppressor of cytokine signaling 3 in cartilage destruction via induction of chondrocyte desensitization toward insulin-like growth factor. Arthritis Rheum. 2006;54:1518-28.

28. de Andres MC, Imagawa K, Hashimoto K, Gonzalez A, Goldring MB, Roach $\mathrm{HI}$, Oreffo RO. Suppressors of cytokine signalling (SOCS) are reduced in osteoarthritis. Biochem Biophys Res Commun. 2011:407:54-9.

29. van de Loo FA, Veenbergen S, van den Brand B, Bennink MB, BlaneyDavidson E, Arntz OJ, van Beuningen HM, van der Kraan PM, van den Berg WB. Enhanced suppressor of cytokine signaling 3 in arthritic cartilage dysregulates human chondrocyte function. Arthritis Rheum. 2012:64:3313-23.

30. Altman R, Asch E, Bloch D, Bole G, Borenstein D, Brandt K, Christy W, Cooke TD, Greenwald R, Hochberg M. Development of criteria for the classification and reporting of osteoarthritis. Classification of osteoarthritis of the knee. Diagnostic and Therapeutic Criteria Committee of the American Rheumatism Association. Arthritis Rheum. 1986;29:1039-49.

31. van Beuningen HM, Stoop $R$, Buma $P$, Takahashi $N$, van der Kraan PM, van den Berg WB. Phenotypic differences in murine chondrocyte cell lines derived from mature articular cartilage. Osteoarthritis Cartilage. 2002;10:977-86.

32. Green LC, Wagner DA, Glogowski J, Skipper PL, Wishnok JS, Tannenbaum SR. Analysis of nitrate, nitrite, and [15 N]nitrate in biological fluids. Anal Biochem. 1982;126:131-8.

33. Livak KJ, Schmittgen TD. Analysis of relative gene expression data using real-time quantitative PCR and the 2(-Delta Delta $C(T))$ method. Methods. 2001:25:402-8.

34. Hovsepian E, Penas F, Siffo S, Mirkin GA, Goren NB. IL-10 inhibits the NFkappaB and ERK/MAPK-mediated production of pro-inflammatory mediators by up-regulation of SOCS-3 in Trypanosoma cruzi-infected cardiomyocytes. PLOS One. 2013;8:e79445.

35. Chhabra JK, Chattopadhyay B, Paul BN. SOCS3 dictates the transition of divergent time-phased events in granulocyte TNF-alpha signaling. Cell Mol Immunol. 2014;11:105-6.

36. Isomäki $P$, Alanära T, Isohanni $P$, Lagerstedt A, Korpela M, Moilanen T, Visakorpi T, Silvennoinen O. The expression of SOCS is altered in rheumatoid arthritis. Rheumatology (Oxford). 2007:46:1538-46.

37. Shouda T, Yoshida T, Hanada T, Wakioka T, Oishi M, Miyoshi K, Komiya S, Kosai K, Hanakawa Y, Hashimoto K, Nagata K, Yoshimura A. Induction of the cytokine signal regulator SOCS3/CIS3 as a therapeutic strategy for treating inflammatory arthritis. J Clin Invest. 2001;108:1781-8.

38. Veenbergen S, Bennink MB, Affandi AJ, Bessis N, Biton J, Arntz OJ, van den Berg WB, van de Loo FA. A pivotal role for antigen-presenting cells overexpressing SOCS3 in controlling invariant NKT cell responses during collagen-induced arthritis. Ann Rheum Dis. 2011;70:2167-75.

39. Wong PK, Egan PJ, Croker BA, O'Donnell K, Sims NA, Drake S, Kiu H, McManus EJ, Alexander WS, Roberts AW, Wicks IP. SOCS-3 negatively regulates innate and adaptive immune mechanisms in acute IL-1dependent inflammatory arthritis. J Clin Invest. 2006;116:1571-81.

40. White CA, Nicola NA. SOCS3: An essential physiological inhibitor of signaling by interleukin-6 and G-CSF family cytokines. JAKSTAT. 2013;2:e25045.

41. Tsuchida Al, Beekhuizen M, 't Hart MC, Radstake TR, Dhert WJ, Saris DB, van Osch GJ, Creemers LB. Cytokine profiles in the joint depend on pathology, but are different between synovial fluid, cartilage tissue and 
cultured chondrocytes. Arthritis Res Ther. 2014;16:441. doi:10.1186/ s13075-014-0441-0.

42. White GE, Cotterill A, Addley MR, Soilleux EJ, Greaves DR. Suppressor of cytokine signalling protein SOCS3 expression is increased at sites of acute and chronic inflammation. J Mol Histol. 2011:42:137-51.

43. Holter K, Wermter AK, Scherag A, Siegfried W, Goldschmidt H, Hebebrand J, Hinney A. Analysis of sequence variations in the suppressor of cytokine signaling (SOCS)-3 gene in extremely obese children and adolescents. BMC Med Genet. 2007:8:21.

44. Toivanen AT, Heliövaara M, Impivaara O, Arokoski JP, Knekt P, Lauren $H$, Kröger H. Obesity, physically demanding work and traumatic knee injury are major risk factors for knee osteoarthritis-a population-based study with a follow-up of 22 years. Rheumatology (Oxford). 2010;49:308-14.

45. Ding C, Parameswaran V, Cicuttini F, Burgess J, Zhai G, Quinn S, Jones G. Association between leptin, body composition, sex and knee cartilage morphology in older adults: the Tasmanian older adult cohort (TASOAC) study. Ann Rheum Dis. 2008:67:1256-61.

46. Ku JH, Lee CK, Joo BS, An BM, Choi SH, Wang TH, Cho HL. Correlation of synovial fluid leptin concentrations with the severity of osteoarthritis. Clin Rheumatol. 2009;28:1431-5.

47. Gandhi R, Takahashi M, Smith H, Rizek R, Mahomed NN. The synovial fluid adiponectin-leptin ratio predicts pain with knee osteoarthritis. Clin Rheumatol. 2010;29:1223-8.

48. Berry PA, Jones SW, Cicuttini FM, Wluka AE, Maciewicz RA. Temporal relationship between serum adipokines, biomarkers of bone and cartilage turnover, and cartilage volume loss in a population with clinical knee osteoarthritis. Arthritis Rheum. 2011;63:700-7.

49. Yusuf E, loan-Facsinay A, Bijsterbosch J, Klein-Wieringa I, Kwekkeboom J, Slagboom PE, Huizinga TW, Kloppenburg M. Association between leptin, adiponectin and resistin and long-term progression of hand osteoarthritis. Ann Rheum Dis. 2011;70:1282-4.

50. de Boer TN, van Spil WE, Huisman AM, Polak AA, Bijlsma JW, Lafeber FP, Mastbergen SC. Serum adipokines in osteoarthritis; comparison with controls and relationship with local parameters of synovial inflammation and cartilage damage. Osteoarthritis Cartilage. 2012;20:846-53.

51. Massengale M, Reichmann WM, Losina E, Solomon DH, Katz JN. The relationship between hand osteoarthritis and serum leptin concentration in participants of the Third National Health and Nutrition Examination Survey. Arthritis Res Ther. 2012:14:R132.

52. Massengale M, Lu B, Pan JJ, Katz JN, Solomon DH. Adipokine hormones and hand osteoarthritis: radiographic severity and pain. PLoS One. 2012;7:e47860.

53. Karvonen-Gutierrez CA, Harlow SD, Mancuso P, Jacobson J, de Leon CFM, Nan B. Association of leptin levels with radiographic knee osteoarthritis among a cohort of midlife women. Arthritis Care Res (Hoboken). 2013;65: 936-44.

54. Karvonen-Gutierrez CA, Harlow SD, Jacobson J, Mancuso P, Jiang Y. The relationship between longitudinal serum leptin measures and measures of magnetic resonance imaging-assessed knee joint damage in a population of mid-life women. Ann Rheum Dis. 2013;73:883-9.

55. Fowler-Brown A, Kim DH, Shi L, Marcantonio E, Wee CC, Shmerling RH, Leveille $S$. The mediating effect of leptin on the relationship between body weight and knee osteoarthritis in older adults. Arthritis Rheumatol. 2015;67: 169-75

56. Griffin TM, Huebner JL, Kraus VB, Guilak F. Extreme obesity due to impaired leptin signaling in mice does not cause knee osteoarthritis. Arthritis Rheum. 2009;60:2935-44.

\section{Submit your next manuscript to BioMed Central and we will help you at every step:}

- We accept pre-submission inquiries

- Our selector tool helps you to find the most relevant journal

- We provide round the clock customer support

- Convenient online submission

- Thorough peer review

- Inclusion in PubMed and all major indexing services

- Maximum visibility for your research

Submit your manuscript at www.biomedcentral.com/submit
) Biomed Central 